\title{
Statistical analysis of the spatial distribution of operons in the transcriptional regulation network of Escherichia coli
}

\author{
P. B. Warren ${ }^{1,2}$ and P. R. ten Wolde $\mathrm{W}^{1,3}$ \\ ${ }^{1}$ FOM Institute for Atomic and Molecular Physics, \\ Kruislaan 407, 1098 SJ Amsterdam, The Netherlands \\ ${ }^{2}$ Unilever R\&D Port Sunlight, Bebington, Wirral, CH63 3JW, UK \\ ${ }^{3}$ Division of Physics and Astronomy, Vrije Universiteit, \\ De Boelelaan 1081, $1081 \mathrm{HV}$ Amsterdam, The Netherlands
}

(Dated: October 16, 2003 art-oct-v5.tex)

\begin{abstract}
We have performed a statistical analysis of the spatial distribution of operons in the transcriptional regulation network of Escherichia coli. The analysis reveals that operons that regulate each other and operons that are coregulated tend to lie next to each other on the genome. Moreover, these pairs of operons tend to be transcribed in diverging directions. This spatial arrangement of operons allows the upstream regulatory regions to interfere with each other. This affords additional regulatory control, as illustrated by a mean-field analysis of a feed-forward loop. Our results suggest that regulatory control can provide a selection pressure that drives operons together in the course of evolution.
\end{abstract}

Most, if not all, organisms can respond and adapt to a changing environment. To this end, they can detect, transmit, and amplify environmental signals, as well as integrate different signals to perform computations analogous to electronic devices. Indeed, all organisms can be considered to be information processing machines. Yet, how the living cell accurately processes information, is still poorly understood. Recent technological developments, however, have made it possible to acquire information on the regulatory architecture of the cell on a massive scale, and extensive databases are now available that catalog biochemical networks. This offers unprecedented possibilities to unravel the design principles by which organisms process information.

The current richness of genomic data surrounding Escherichia coli makes it no doubt one of the best characterized of all living organisms. The condensation of genes into operons and the organization of operons into the transcriptional regulation network are now well mapped, and this information has been used to investigate generic features such as the appearance of motifs in the transcriptional regulation network 1]. Here, we present a study of the spatial organization of operons in the transcriptional regulation network of $E$. coli. Our analysis of the spatial distribution of operons provides two distinct advantages over previous studies on the spatial distribution of genes 2, 3, 4, 5, 6, 7, 8] : firstly, it excludes correlations from genes that belong to the same operon. Secondly, and more importantly, by focusing on the higher-level organisation of operons into the transcriptional regulation network, the analysis allows us to elucidate spatial correlations associated with regulatory control, for in- stance, by identifying coregulated pairs of operons that are adjacent on the DNA.

We find that there is a marked tendency for operons that are related to each other in the transcriptional regulation network to be nearest neighbours, compared to networks in which operons are randomly assigned positions on the DNA. Furthermore, the separations between neighbour pairs have a strong bias towards short distances, which is most pronounced for pairs that are transcribed in diverging directions. In fact, our analysis identifies a new, spatial network motif that consists of pairs of overlapping operons - operons of which the upstream regulatory domains overlap.

Several mechanisms could give rise to the observed distributions. The strong bias towards short separations could be a result of the mechanisms by which genes and connections between genes arise and disappear during evolution. In contrast, it is also conceivable that there is a functional benefit for having certain operons close to each other. This would lead to a selection pressure for shorter separations between certain operons. We do not investigate these scenarios in detail here, but our data does support the latter scenario for the diverging neighbour pairs. In particular, we examine a network motif that has been identified by our statistical analysis: a feed-forward loop in which the 'downstream' operons are transcribed in diverging directions. The analysis shows that overlapping regulatory domains for these operons can strongly enhance the response of the network. Hence, our results suggest that regulatory control can provide an evolutionary driving force for the formation of overlapping operons.

\section{Methods}

Our starting point is the transcriptional regulation network data compiled by Shen-Orr et al for their motif analysis [1]. We have annotated their list of operons with the start- and end-points of the coding regions, extracted from the various databases of genomic information for $E$. coli [9, 10]. We work with coding regions rather than promoters because the former are easy to identify and the distance between a promoter and the start of the coding region is small compared to the typical distances we consider here. The transcriptional regulation network contains 404 operons with 558 links.

We focus on the statistics of the pair separations be- 


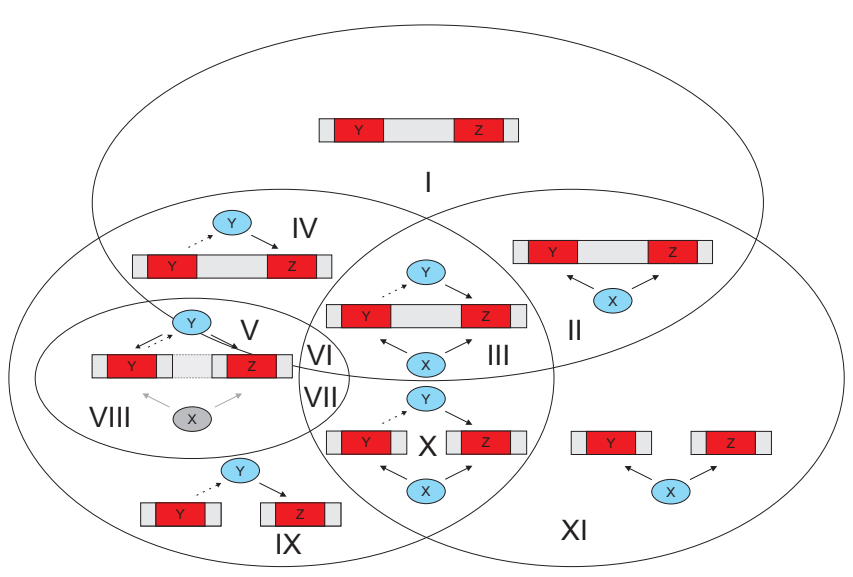

FIG. 1: Schematic overview of the sets of network motifs studied in this paper. The full set is given by the ensemble of all possible operon pairs. We have split the full set into the following subsets: the ensemble of nearest neighbour pairs (NN), corresponding to regions I-VI; the set of pairs that are connected in the transcriptional regulation network (TRN), corresponding to regions III-X; the set of pairs that are coregulated by a distinct third operon (CR), corresponding to II+III+VI+VII+X+XI; the set of autoregulated pairs (AR), which is a proper subset of the TRN set (regions VVIII; here, protein $\mathrm{X}$ only regulates operon $\mathrm{Y}$ and $\mathrm{Z}$ in regions VI and VII). The union of TRN and CR sets (subsets $\mathrm{III}+\mathrm{VI}+\mathrm{VII}+\mathrm{X}$ ) corresponds to feed-forward loops (FFLs), a network motif identified by Shen-Orr et al [1].

tween operons which are related in the transcriptional regulation network by various definitions (see below), and on pairs of neighboring operons on the genome. We define the pair separation to be the distance along the DNA between the coding regions (an alternative definition as the distance between the starting points for transcription was explored with similar results). One basic tool is the cumulative distribution function $F(s)=\int_{0}^{s} d s^{\prime} P\left(s^{\prime}\right)$, where $P(s) d s$ is the probability that the distance between two operons along the DNA has a value between $s$ and $s+d s$. The cumulative distribution function $F(s)$ is used in preference to the probability distribution function $P(s)$ because it is readily visualized even for sparse data sets, and does not need to be corrected if a logarithmic axis is used for $s$.

We base our analysis on the operon pairs in three overlapping sets: pairs of operons that are nearest neighbours (NN) on the DNA, pairs of operons in the transcriptional regulation network (TRN), and pairs of operons that are coregulated (CR) by a third operon. In addition, we have also considered autoregulated (AR) pairs which are TRN pairs for which the controlling operon also regulates itself. The different sets and subsets are schematically indicated in Fig. 11 and the sizes of the sets in Table 1 .

In order to determine the statistical significance of the different quantities for the $E$. coli network, we have calculated the corresponding expectation values for an ensemble of random networks. Since we are primarily interested in the network motifs that arise due to the spatial
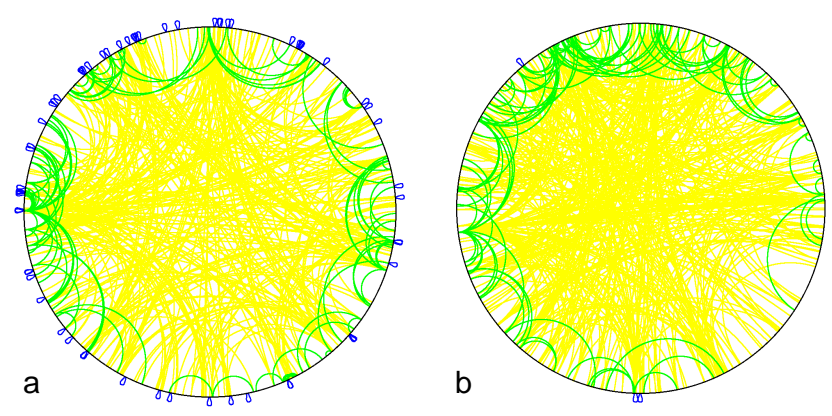

FIG. 2: The transcriptional regulation network of E. coli shown as links between operons on the genome. Maps are shown for (a) the real E. coli network and (b) a representative 'randomised' network with the same topology, but with a random permutation of the positions of the operons. The color code is: blue: $s<10 \mathrm{kbp}$; green: $10 \mathrm{kbp}<s<500 \mathrm{kbp}$; yellow: $s>500 \mathrm{kbp}$. Note the much greater prevalence of the 'short' distances in the real map (a) compared to the randomised map (b).

organisation of the network and not in those that are a consequence of the topology of the network (which have already been identified by Shen-Orr et al [1]), we define a random network to be a network with a connectivity of that of the $E$. coli network, but with a random assignment of operon positions and orientations on the genome. Hence, not only the NN set of the $E$. coli network and a random network are equal in size, also the sizes of the TRN, CR and AR sets in the E. coli network equal those in a random network, as the sizes of these sets are determined by the topology of the network. In contrast, the sizes of the unions corresponding to regions II-VI (see Fig. (1) differ between the E. coli network and a random network, since they are determined by the spatial distribution of the operons. For the E. coli network, we can directly obtain the sizes of the respective unions from the various databases [9, 10]. For the ensemble of random networks, we have calculated the expectation value for the number of pairs, $M_{\alpha}$, in the union formed by the overlap of the set of nearest neighbour pairs with set $\alpha$ (be it set TRN, AR or CR), using $M_{\alpha}=p N_{\alpha}$, where $p$ is the probability that a randomly chosen pair is, in fact, a nearest neighbour pair and $N_{\alpha}$ is the number of pairs in set $\alpha$. We have verified these calculations by generating random networks and computing the quantities directly. The $P$-values reported are probabilities of finding a subset in the random network of at least the size as observed in E. coli, computed using the same statistical model.

\section{Results}

\section{Transcriptional Regulation Network}

In fig. 2 we compare a 'map' of the real transcriptional regulation network to a map of a randomised version of the network; the random network has been obtained by randomly permuting the positions of the operons on the DNA, thus preserving the topology of the network. It is 


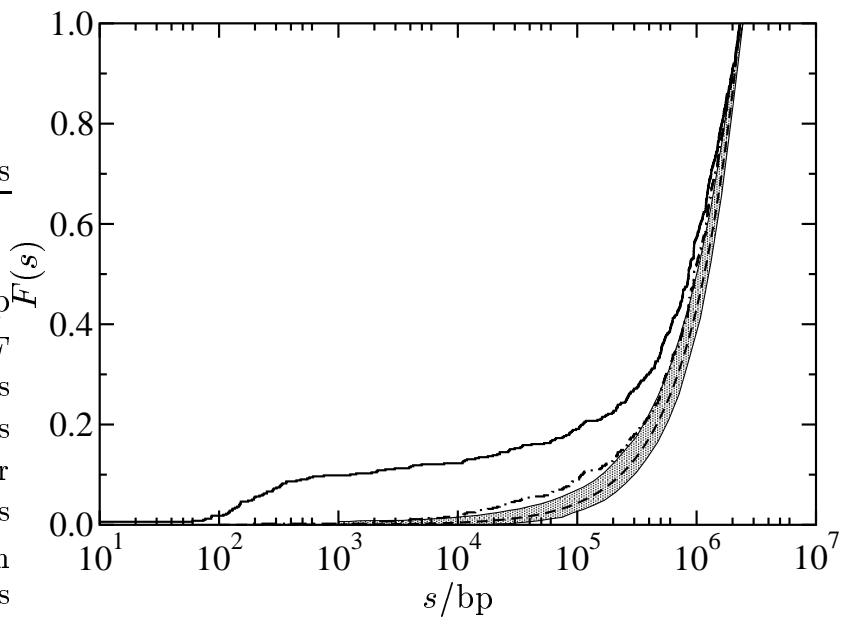

FIG. 3: The cumulative probability distribution $F(s)$ for the distances along the DNA between operons in the transcriptional regulatory network (TRN) of E. coli (solid line). The dashed line corresponds to the average of an ensemble of networks that have been obtained by randomly permuting the positions of the operons, thus preserving the network topology; the grey area denotes the $98 \%$ confidence regime. The dashed line is indistinguishable from the distribution function that is expected for a random network, which is given by $F(s)=s /\left(L_{\mathrm{DNA}} / 2\right)$. Note the significant $10-15 \%$ fraction with $s \lesssim 1 \mathrm{kbp}$ for the $E$. coli network. The dotted-dashed line corresponds to the TRN pairs that are not nearest neighbours. The mean lengths of genes and operons in the data set are $1.0 \mathrm{kbp}$ and $2.1 \mathrm{kbp}$, respectively.

seen that the real network exhibits a larger number of 'short' (blue) links and a smaller number of 'long' (yellow) links, as compared to the random network. This indicates that operons that regulate each other tend to lie closer to each other than can be expected for a random network. We can quantify this by calculating the distribution functions for the distances between the network pairs. Fig. [3]shows the cumulative disitribution functions for the E. coli network and for the ensemble of random networks. For the ensemble of random networks, we expect that the separation between network pairs is uniformly distributed between zero and $L_{\mathrm{DNA}} / 2=2.3 \mathrm{Mbp}$, where $L_{\mathrm{DNA}}=4.64 \mathrm{Mbp}$ is the total length of the DNA. Fig. 3) shows that this is indeed the case. For the real network, however, we find marked deviations from a uniform distribution. It is seen that up to $\approx 200 \mathrm{bp}$, the cumulative distribution function is close to zero; this lower cut-off reflects the typical sizes of promoter regions for operons. However, after $\approx 200 \mathrm{bp}$ the cumulative distribution function of the $E$. coli network sharply increases by some $10-15 \%$, until it follows a nearly uniform distribution from $\approx 1 \mathrm{kbp}$ upwards.

What is the origin of the pronounced increase in $F(s)$ at around $200 \mathrm{bp?}$ Do operons that are linked in the transcriptional regulation network tend to be nearest neighbors? Table \ shows that is indeed the case. In E. coli, 55 out of the 497 transcriptional regulation network pairs are nearest neighbours, as compared to the average of 2.5 in the ensemble of random networks. Moreover, these (NN,TRN) pairs tend to lie very close to each other: out of the 55 (NN,TRN) pairs, 44 are located within $500 \mathrm{bp}$ from each other, which is much smaller than the mean spacing of $9.6 \mathrm{kbp}$ between operons. Fig. [3 also shows that for the ensemble of transcriptional regulation network pairs that are not nearest neighbours, the cumulative distribution function is much closer to the average of the ensemble of random networks. This establishes that the bias towards short distances in the transcriptional regulation network is due to the tendency of network pairs to be nearest neighbours.

\section{Neighboring operons on the DNA}

The results on the separation statistics for the transcriptional regulation network motivated us to examine the nearest neighbour pairs in more detail. We did not only include pairs that constitute links in the transcriptional regulation network (regions III-VI) in Fig. 1] but also pairs that are coregulated by a common transcription factor (regions II,III and VI in Fig. (1).

Table 【 shows the sizes of the unions formed by the overlap of, on the hand, the set of nearest neighbour $(\mathrm{NN})$ pairs, and, on the other hand, the sets of (autoregulatory) network pairs and coregulated pairs, respectively. It is seen that these unions are significantly larger than the corresponding unions in the ensemble of random networks. Moreover, region I, corresponding to the ensemble of nearest neighbours that are neither coregulated nor form a link in the transcriptional regulation network, is smaller than the corresponding region in the ensemble of random networks (data not shown). Clearly, both operons that regulate each other and operons that are coregulated by a common transcription factor, tend to be nearest neighbours on the DNA.

A spatial arrangement of operons in which adjacent operons are transcribed in diverging directions, allows the upstream regulatory regions to overlap. As we discuss in more detail below, this affords additional regulatory control. We therefore addressed two questions: 1) Do operons that are each other's nearest neighbours tend to be transcribed in diverging directions? 2) Do these nearest neighbours tend to lie relatively close to each other?

Table \ shows the statistics for pairs of operons broken down according to the relative direction of transcription. There are three classes: 'tandem' (both operons are transcribed in a common direction), 'converging' and 'diverging'. If the three classes of orientation were populated in a random manner, one would expect the ratio converging : diverging : tandem $=1: 1: 2$. Our analysis reveals that in region I and in regions VII - XI, the three classes are indeed populated in a nearly random manner (data not shown). In contrast, the orientation statistics for adjacent operons that are either coregulated or form a (autoregulatory) link in the transcriptional regulation network, are markedly different. These pairs tend to be transcribed in diverging directions. Moreover, this effect 


\begin{tabular}{|c|c|c|c|c|c|c|c|}
\hline \multirow[b]{2}{*}{ Set } & \multirow[b]{2}{*}{ Orientation } & \multicolumn{3}{|c|}{ all pairs } & \multicolumn{3}{|c|}{ pairs $(s<500 \mathrm{bp})$} \\
\hline & & E. coli & Random & $P$-value & E. coli & Random & $P$-value \\
\hline Regulation Network (TRN) & & 497 & & & 45 & & \\
\hline Coregulated (CR) & & 4362 & & & 27 & & \\
\hline Autoregulated (AR) & & 318 & & & 23 & & \\
\hline TRN \& CR (ie FFls) & & 42 & & & 6 & & \\
\hline $\mathrm{AR} \& \mathrm{CR}$ & & 24 & & & 5 & & \\
\hline $\begin{array}{l}\text { Nearest Neighbours (NN) } \\
(\mathrm{I}-\mathrm{VI})\end{array}$ & $\begin{array}{l}\text { Diverging } \\
\text { Converging } \\
\text { Tandem }\end{array}$ & $\begin{array}{l}103 \\
105 \\
188\end{array}$ & & & $\begin{array}{l}45 \\
13 \\
42\end{array}$ & $\begin{array}{l}4.8 \\
4.8 \\
9.6\end{array}$ & $\begin{array}{l}10^{-28} \\
10^{-3} \\
10^{-14}\end{array}$ \\
\hline $\begin{array}{l}\text { TRN \& NN } \\
(\text { III-VI) }\end{array}$ & $\begin{array}{l}\text { Diverging } \\
\text { Converging } \\
\text { Tandem }\end{array}$ & $\begin{array}{r}33 \\
3 \\
19\end{array}$ & $\begin{array}{l}0.63 \\
0.63 \\
1.3\end{array}$ & $\begin{array}{l}10^{-44} \\
10^{-2} \\
10^{-16}\end{array}$ & $\begin{array}{r}27 \\
2 \\
15\end{array}$ & $\begin{array}{l}0.031 \\
0.031 \\
0.061\end{array}$ & $\begin{array}{l}10^{-69} \\
10^{-4} \\
10^{-31}\end{array}$ \\
\hline $\begin{array}{l}\mathrm{CR} \& \mathrm{NN} \\
(\mathrm{II}+\mathrm{III}+\mathrm{VI})\end{array}$ & $\begin{array}{l}\text { Diverging } \\
\text { Converging } \\
\text { Tandem }\end{array}$ & $\begin{array}{l}22 \\
12 \\
26\end{array}$ & $\begin{array}{r}5.5 \\
5.5 \\
11 .\end{array}$ & $\begin{array}{l}10^{-7} \\
10^{-2} \\
10^{-4}\end{array}$ & $\begin{array}{r}15 \\
2 \\
8\end{array}$ & $\begin{array}{l}0.27 \\
0.27 \\
0.54\end{array}$ & $\begin{array}{l}10^{-21} \\
10^{-2} \\
10^{-7}\end{array}$ \\
\hline $\begin{array}{l}\mathrm{AR} \& \mathrm{NN} \\
(\mathrm{V}+\mathrm{VI})\end{array}$ & $\begin{array}{l}\text { Diverging } \\
\text { Converging } \\
\text { Tandem }\end{array}$ & $\begin{array}{r}21 \\
1 \\
5\end{array}$ & $\begin{array}{l}0.40 \\
0.40 \\
0.81\end{array}$ & $\begin{array}{l}10^{-28} \\
0.3 \\
10^{-3}\end{array}$ & $\begin{array}{r}18 \\
1 \\
4\end{array}$ & $\begin{array}{l}0.020 \\
0.020 \\
0.040\end{array}$ & $\begin{array}{l}10^{-47} \\
10^{-2} \\
10^{-7}\end{array}$ \\
\hline $\begin{array}{l}\mathrm{TRN} \& \mathrm{CR} \& \mathrm{NN} \\
(\mathrm{III}+\mathrm{VI})\end{array}$ & $\begin{array}{l}\text { Diverging } \\
\text { Converging } \\
\text { Tandem }\end{array}$ & $\begin{array}{l}6 \\
1 \\
1\end{array}$ & $\begin{array}{l}0.054 \\
0.054 \\
0.11\end{array}$ & $\begin{array}{l}10^{-11} \\
10^{-2} \\
0.1\end{array}$ & $\begin{array}{l}4 \\
1 \\
1\end{array}$ & $\begin{array}{l}0.0026 \\
0.0026 \\
0.0052\end{array}$ & $\begin{array}{l}10^{-12} \\
10^{-3} \\
10^{-2}\end{array}$ \\
\hline
\end{tabular}

TABLE I: Sizes of (sub)sets shown in Fig. 1for E. coli; the roman numerals between brackets refer to the regions in Fig. 1 The quantities in the 'Random' column refer to averages in the ensemble of random networks as defined in Method. The $P$-values are probabilities that a quantity of at least the size as observed in the E. coli network can be found in a random network. The TRN \& CR \& NN (III+VI) is identical to the AR \& CR \& NN set (VI) (not shown) apart from the additional presence of one converging operon pair.

is most pronounced if the operons lie very close to each other on the DNA. Table \ shows that coregulated and (autoregulatory) network pairs that lie less than $500 \mathrm{bp}$ apart from each other, are predominantly transcribed in diverging directions.

To answer the second question, we have calculated the separation-distribution function $F(s)$ for nearest neighbour pairs. If operons were distributed at random on the genome, one would expect the separation statistics to follow a Poisson distribution; this corresponds to a model in statistical physics known as a Tonks gas [11]. Fig. 4 however, shows that the E. coli network exhibits strong deviations from Poisson statistics. In particular, it shows that a large number of links are distinctively short. This is most striking for operons that are transcribed in diverging directions, although it is also noticeable for operons that are transcribed in a common direction. About $45 \%$ of diverging pairs and $20 \%$ of tandem pairs are closer than $500 \mathrm{bp}$, and these fractions are much higher than would be expected for a random network (see also Table 【). It appears that the transcriptional regulation network of $E$. coli has a large fraction of adjoining operons. Importantly, Fig. 4 $\mathrm{b}$ indicates that most of these adjoining operons are, indeed, operons that either regulate each other or are controlled by a common transcription factor.
As mentioned above, an arrangement in which neighboring operons are transcribed in diverging directions, allows the operator regions to interfere with each other. The occurrence of diverging neighbour pairs with operator interference can be assessed by a careful examination of the EcoCyc database [9, 10] (for details see supplementary information). Of the 45 diverging neighbour pairs with $s<500$ bp (see Table【), 20 operon pairs have operator interference (there are, in fact, 3 examples of operator interference with $s>500 \mathrm{bp}$ ); 10 do not; and for the remaining 15 , there is insufficient information on the promoter/operator regions to decide. We conclude that the presence of operator interference provides a major part of the explanation for the strong bias towards small separations for diverging pairs.

\section{Discussion}

The principal findings of our statistical analysis are: 1) pairs of operons that regulate each other and pairs of operons that are coregulated tend to be nearest neighbours; 2) these nearest neighbours tend to be transcribed in diverging directions; 3 ) the nearest neighbours' separation statistics is strongly biased towards short distances. What could be the origin of this behavior? Two distinct scenarios can give rise to the observed separation statistics. In the first, the bias is a result of the mechanisms 

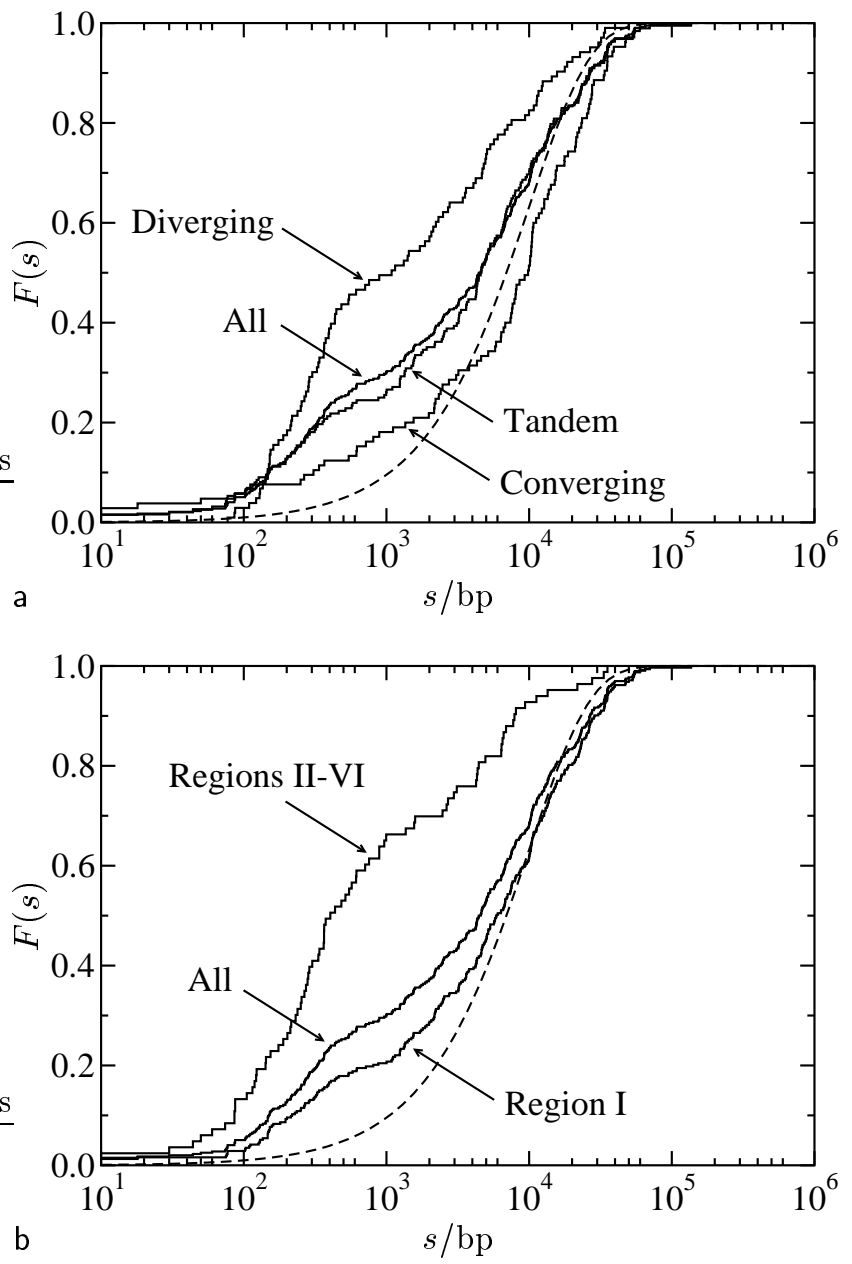

FIG. 4: The cumulative distribution functions $F(s)$ for nearest neighbour pairs (NN pairs) in the E. coli network: (a) split by relative orientation; and (b) split according to whether the neighbour pair is coregulated or in the transcriptional regulation network (regions II-VI), or not (region I). The dashed line is given by a Poisson distribution, $F(s)=1-$ $\exp \left[-s / L_{\text {rand }}\right]$, where $L_{\text {rand }}=9.6 \mathrm{kbp}$ is the mean spacing between operons.

by which new operons and new links between operons emerge and disappear in the course of evolution. In the second, it is a consequence of a functional benefit for having short separations between certain pairs of operons; in this scenario, there is a selection pressure towards shorter distances. It seems hard, if not impossible, to disentangle both mechanisms, although, in principle, there is a clear difference between the two. In the former scenario, newly emerged operons will drift apart and, as a function of time, the distance between them will increase. In contrast, in the latter scenario, a selection pressure will drive and keep the operons together.

Still, the question remains why it could be beneficial to have pairs of operons together. It is believed that in prokaryotes transcription and translation take place simultaneously (see Fig. 1.2 in Wagner [12]). It seems ad-

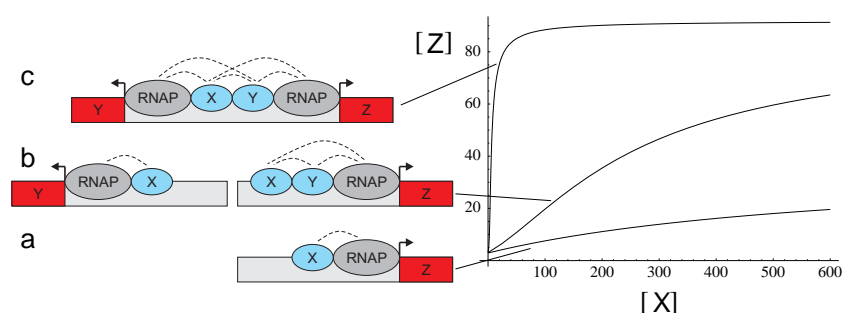

FIG. 5: Response of FFLs as a function of the spatial arrangement of the regulatory elements. The transcription factors $\mathrm{X}$ and $\mathrm{Y}$ coherently regulate the expression of operon $\mathrm{Z}$; a dashed line indicates a weak cooperative interaction of $\approx 3 k_{B} T$, which corresponds to a cooperativity factor $\omega \approx 20$; RNAP denotes the enzyme RNA polymerase. On the left the different structures: a the expression of gene $\mathrm{Z}$ is activated by the transcription factor X only; $\mathbf{b}$ a 'classical' FFL; c a FFL in which the operator regions overlap. On the right, the concentration in $\mathrm{nM}$ of the expressed protein $\mathrm{Z}$ as a function of that of the transcription factor X; the inducer for transcription factor $\mathrm{Y}$ is assumed to be present at saturating concentrations [13]. It is seen that a FFL can act as an amplifier and that overlapping operons can significantly enhance the performance of the amplifier.

vantageous to have transcription factors expressed close to the locus at which they are supposed to act. Furthermore, two operons can be topologically coupled via the interplay between transcription and supercoiling 12 . This can lead to additional regulatory control, but only if the distance between the operons is covered by the twin-supercoiled domains.

Perhaps the most interesting case concerns the prevalence for short distances amongst neighboring operons that are transcribed in diverging directions. This spatial arrangement of operons offers the possibility that the operator regions interfere with each other. This can provide an extra layer of regulatory control. Just as the existence of operons provides for correlated gene expression, interference between the regulatory regions for a pair of diverging operons affords additional opportunities for correlated or anticorrelated expression of operons.

One of the simplest regulatory constructs is a genetic switch consisting of two operons that mutually repress each other, and elsewhere we will publish a detailed analysis of the consequences of correlated and anticorrelated operon expression for the stability of such toggle switches [14]. Toggle switches are not a statistically significant motif in the transcriptional regulation network of $E$. coli though, and to demonstrate the effect we turn to an example based around a feed-forward loop (FFL). Shen-Orr et al [1] demonstrated that FFLs are important computational elements of the $E$. coli network and it is believed that they can perform a variety of computational tasks in a regulatory circuit. It is believed that they can filter transient signals [1, 13], act as sign-sensitive accelerators or sign-sensitive delays [13], or act as an amplifier, in which the activity of the gene at the top of the loop is amplified at the ultimate target gene [13, 15]. 
Our statistical analysis has revealed that in FFLs the downstream operons tend to overlap (see Table【). In order to investigate the role of overlapping operons in FFLs, we have performed a mean-field analysis of FFLs in which transcription factors $\mathrm{X}$ and $\mathrm{Y}$ coherently activate the expression of operon Z [13] (see Fig. [5] and supplementary material for details). Fig. [5 shows that overlapping operons can strongly enhance the sharpness of the response. In contrast to the general scheme (Fig. [5), the gene regualtory proteins $\mathrm{X}$ and $\mathrm{Y}$ can simultaneously activate gene $\mathrm{Y}$ and gene $\mathrm{Z}$ in the overlapping operon scenario (Fig. 55). This allows for extra cooperativity, which in turn leads to a sharper response. It would seem that the capacity to generate a strong reponse can confer a competitive advantage to the organism in a number of cases, such as in the repression of sugar-uptake systems in response to glucose. Hence, our results suggest that regulatory control can provide a selection pressure that drives operons together in the course of evolution.

\section{Acknowledgements}

We thank Marileen Dogterom, Daan Frenkel, Sander Tans and Conrad Woldringh for useful discussions and a careful reading of the manuscript. The work is supported by the Amsterdam Centre for Computational Science (ACCS). This work is part of the research program of the 'Stichting voor Fundamenteel Onderzoek der Materie (FOM)', which is financially supported by the 'Nederlandse organisatie voor Wetenschappelijk Onderzoek (NWO)'.
[1] Shen-Orr, S. S, Milo, R, Mangan, S, \& Alon, U. (2002) Nature Genetics 31, 64-68.

[2] Tamames, J, Casari, G, Ouzounis, C, \& Valencia, A. (1997) J. Mol. Evol. 44, 66-73.

[3] Huynen, M. A \& Bork, P. (1998) Proc. Natl. Acad. Sci. USA 95, 5849-5856.

[4] Overbeek, R, Fonstein, M, D'Souza, M, Push, G. D, \& Maltsev, N. (1999) Proc. Natl. Acad. Sci. USA 96, 28962901.

[5] Snel, B, Lehmann, G, Bork, P, \& Huynen, M. A. (2000) Nucl. Acids. Res. 28, 3442-3444.

[6] Salgado, H, Moreno-Hagelsieb, G, Smith, T. F, \& Collado-Vides, J. (2000) Proc. Natl. Acad. Sci. USA 97, 6652-6657.

[7] de Daruvar, A, Collado-Vides, J, \& Valencia, A. (2002) J. Mol. Evol. 55, 211-221.

[8] Audit, B \& Ouzounis, C. A. (2003) J. Mol. Biol. 332, 617-633.

[9] To find genes, we used the databases on www.ncbi.nlm.nih.gov, www.embl-heidelberg.de, and version M52 of the genome data for $E$. coli K12 strain MG1655 from www.genome.wisc.edu. We also consulted the EcoCyc regulation database at www.ecocyc.org 10]. The procedures leave 19 operons from the Shen-Orr et al dataset with unresolved positions: 16 contain genes which are not in the current databases, and 3 contain genes which are transcribed in opposed directions. The 19 problematic operons were dropped from the network. One new link from the EcoCyc database was added.

[10] Karp, P. D, M. Riley, M. S, Paulsen, I. T, Collado-Vides, J, S. M. Paley, A. P.-T, Bonavides, C, \& Gama-Castro, S. (2002) Nucl. Acids Res. 30, 56-58.

[11] Lieb, E. H \& Mattis, D. C. (1966) Mathematical Physics in One Dimension. (Academic Press, New York).

[12] Wagner, R. (2000) Transcription regulation in prokaryotes. (OUP, Oxford).

[13] Mangan, S \& Alon, U. (2003) Proc. Natl. Acad. Sci. USA 100, 11980-11985.

[14] P. B. Warren and P. R. ten Wolde, to be submitted.

[15] Lee et al. (2002) Science 298, 799-804.

[16] Shea, M. A \& Ackers, G. K. (1985) J. Mol. Biol. 181, 211-230.

[17] Buchler, N. E, Gerland, U, \& Hwa, T. (2003) Proc. Natl. Acad. Sci. USA 100, 5136-5141.

[18] Ptashne, M \& Gann, A. (1997) Nature 386, 569-577.

[19] Ptashne, M \& Gann, A. (2002) Genes and signals. (Cold Spring Harbor Laboratory Press, New York). 
Supplementary material - overlapping operons

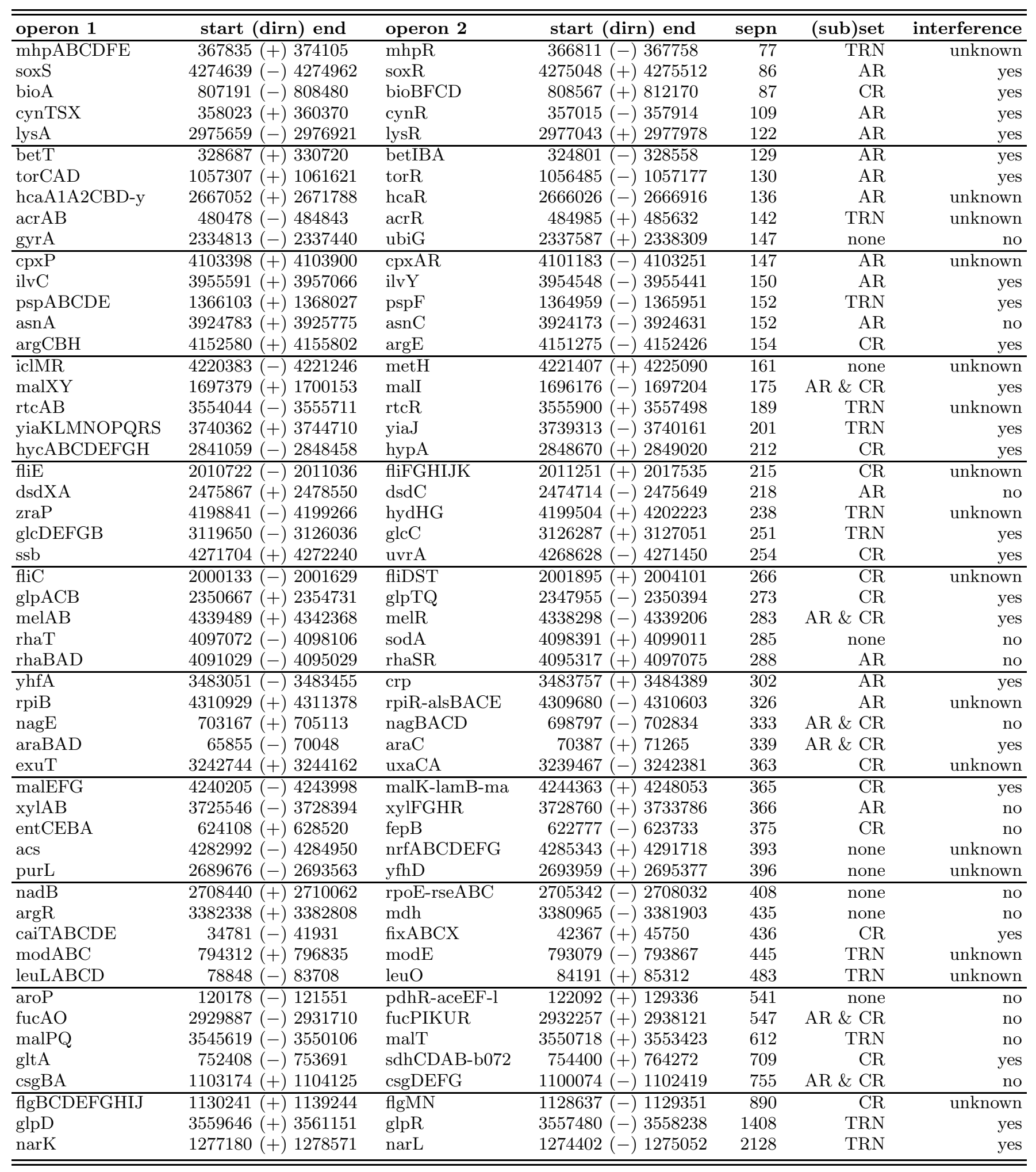

Included are all diverging neighbour pairs with less than $1 \mathrm{kbp}$ separation between coding regions, or with detected interference between operator regions if separation is greater than $1 \mathrm{kbp}$. Operon names have been truncated to 12 characters to save space. All the AR \& CR pairs are also examples of 'downstream' operons in feed-forward loops. For TRN and AR pairs, operon 1 is the controlled operon and operon 2 is the controlling operon. 


\section{Supplementary material - feed-forward loops}

We follow the approach of Shea and Ackers 16 and Buchler et al. 17] in modelling gene expression. The approach relies on the idea of "regulated recruitment" 18, 19]: gene regulatory proteins control gene expression by modulating the probability $P$ that the enzyme RNA polymerase is bound to the DNA; if the RNA polymerase is bound, then it is assumed that gene expression occurs at a fixed rate $\beta$. The macroscopic rate equation for the synthesis and degradation of a protein $\mathrm{Z}$ is thus given by:

$$
\frac{\mathrm{d}[\mathrm{Z}]}{\mathrm{dt}}=\beta P_{\mathrm{Z}}-\mu[\mathrm{Z}]
$$

Here $[\mathrm{Z}]$ is the concentration of protein $\mathrm{Z}, P_{\mathrm{Z}}$ is the probability that the RNA polymerase is bound to the promoter for gene $\mathrm{Z}$ and $\mu$ is the degradation rate of the protein. The probability $P_{\mathrm{Z}}$ that a RNA polymerase is bound to the promoter of gene $\mathrm{Z}$ is given by

$$
P_{\mathrm{Z}}=\frac{Z_{\mathrm{Z}, \mathrm{on}}}{Z_{\mathrm{Z}, \mathrm{off}}+Z_{\mathrm{Z}, \mathrm{on}}}
$$

Here $Z_{\mathrm{Z} \text {,on }}$ and $Z_{\mathrm{Z} \text {,off }}$ are the partition functions for the system with the RNA polymerase bound and not bound to the promoter of gene Z, respectively. Following Buchler et al. [17], we characterize the interaction between a pair of proteins - a protein being either a RNA polymerase or a transcription factor - by a cooperativity factor $\omega$. A weak glue-like interaction of $3 k_{B} T \approx 2 \mathrm{kcal} / \mathrm{mol}$ is assumed [17, 18, 19], which corresponds to a cooperativity factor $\omega \approx 20$. Furhermore, we assume that if gene expression is controlled by two gene regulatory proteins, the RNA polymerase can contact both proteins simultaneously. We have also considered the independent interaction model, in which the RNA polymerase can only interact with one gene regulatory protein at the time 17]. We obtained similar conclusions for the two models.

We now consider the structures shown in Fig. [6 of the supplementary material.

\section{Structure a:}

$$
\begin{aligned}
Z_{\mathrm{Z}, \text { off }} & =1+[\mathrm{X}] / K_{\mathrm{X}} \\
Z_{\mathrm{Z}, \text { on }} & =[\mathrm{RNAP}] / K_{\mathrm{RNAP}}\left(1+\omega[\mathrm{X}] / K_{\mathrm{X}}\right)
\end{aligned}
$$

\section{Structure b:}

$$
\begin{aligned}
Z_{\mathrm{Y}, \text { off }} & =1+[\mathrm{X}] / K_{\mathrm{X}} \\
\mathrm{Z}_{\mathrm{Y}, \text { on }} & =[\mathrm{RNAP}] / K_{\mathrm{RNAP}}\left(1+\omega[\mathrm{X}] / K_{\mathrm{X}}\right) \\
Z_{\mathrm{Z}, \text { off }} & =1+[\mathrm{X}] / K_{\mathrm{X}}+[\mathrm{Y}] / K_{\mathrm{Y}}+\omega[\mathrm{X}][\mathrm{Y}] /\left(K_{\mathrm{X}} K_{\mathrm{Y}}\right) \\
Z_{\mathrm{Z}, \text { on }} & =[\mathrm{RNAP}] / K_{\mathrm{RNAP}}\left(1+\omega[\mathrm{X}] / K_{\mathrm{X}}+\omega[\mathrm{Y}] / K_{\mathrm{Y}}+\omega^{3}[\mathrm{X}][\mathrm{Y}] /\left(K_{\mathrm{X}} K_{\mathrm{Y}}\right)\right) .
\end{aligned}
$$

\section{Structure c:}

$$
\begin{aligned}
Z_{\mathrm{Y}, \text { off }} & =1+[\mathrm{X}] / K_{\mathrm{X}}+[\mathrm{Y}] / K_{\mathrm{Y}}+\omega[\mathrm{X}][\mathrm{Y}] /\left(K_{\mathrm{X}} K_{\mathrm{Y}}\right) \\
\mathrm{Z}_{\mathrm{Y}, \text { on }} & =[\mathrm{RNAP}] / K_{\mathrm{RNAP}}\left(1+\omega[\mathrm{X}] / K_{\mathrm{X}}+\omega[\mathrm{Y}] / K_{\mathrm{Y}}+\omega^{3}[\mathrm{X}][\mathrm{Y}] /\left(K_{\mathrm{X}} K_{\mathrm{Y}}\right)\right) \\
Z_{\mathrm{Z}, \text { off }} & =Z_{\mathrm{Y}, \text { off }} \\
Z_{Z, \text { on }} & =Z_{\mathrm{Y}, \text { on }}
\end{aligned}
$$

It is seen that the expression of $\mathrm{Y}$ depends on the concentration of $\mathrm{Y}$. This means that in order to obtain the concentration of $\mathrm{Y}$, we have to solve a quadratic equation in $[\mathrm{Y}]$. This structure is included to show the effect of the autoregulatory loop on $\mathrm{Y}$ - the transcription factor $\mathrm{Y}$ interacts with the RNA polymerase bound to the promoter for gene Y.

\section{Structure d:}

$$
\begin{aligned}
Z_{\mathrm{Y}, \text { off }}= & 1+[\mathrm{X}] / K_{\mathrm{X}}+[\mathrm{Y}] / K_{\mathrm{Y}}+\omega[\mathrm{X}][\mathrm{Y}] /\left(K_{\mathrm{X}} K_{\mathrm{Y}}\right)+[\mathrm{RNAP}] / K_{\mathrm{RNAP}}+\omega[\mathrm{X}][\mathrm{RNAP}] /\left(K_{\mathrm{X}} K_{\mathrm{RNAP}}\right) \\
& +\omega[\mathrm{Y}][\mathrm{RNAP}] /\left(K_{\mathrm{Y}} K_{\mathrm{RNAP}}\right)+\omega^{3}[\mathrm{X}][\mathrm{Y}][\mathrm{RNAP}] /\left(K_{\mathrm{X}} K_{\mathrm{Y}} K_{\mathrm{RNAP}}\right) \\
Z_{\mathrm{Y}, \text { on }}= & {[\mathrm{RNAP}] / K_{\mathrm{RNAP}}\left(1+\omega[\mathrm{X}] / K_{\mathrm{X}}+\omega[\mathrm{Y}] / K_{\mathrm{Y}}+[\mathrm{RNAP}] / K_{\mathrm{RNAP}}+\right.} \\
& \omega^{3}[\mathrm{X}][\mathrm{Y}] /\left(K_{\mathrm{X}} K_{\mathrm{Y}}\right)+\omega^{2}[\mathrm{X}][\mathrm{RNAP}] /\left(K_{\mathrm{X}} K_{\mathrm{RNAP}}\right)+\omega^{2}[\mathrm{Y}][\mathrm{RNAP}] /\left(K_{\mathrm{Y}} K_{\mathrm{RNAP}}\right) \\
& \left.+\omega^{5}[\mathrm{X}][\mathrm{Y}][\mathrm{RNAP}] /\left(K_{\mathrm{X}} K_{\mathrm{Y}} K_{\mathrm{RNAP}}\right)\right) \\
Z_{\mathrm{Z}, \text { off }}= & Z_{\mathrm{Y}, \text { off }} \\
Z_{\mathrm{Z}, \text { on }}= & Z_{\mathrm{Y}, \text { on }}
\end{aligned}
$$




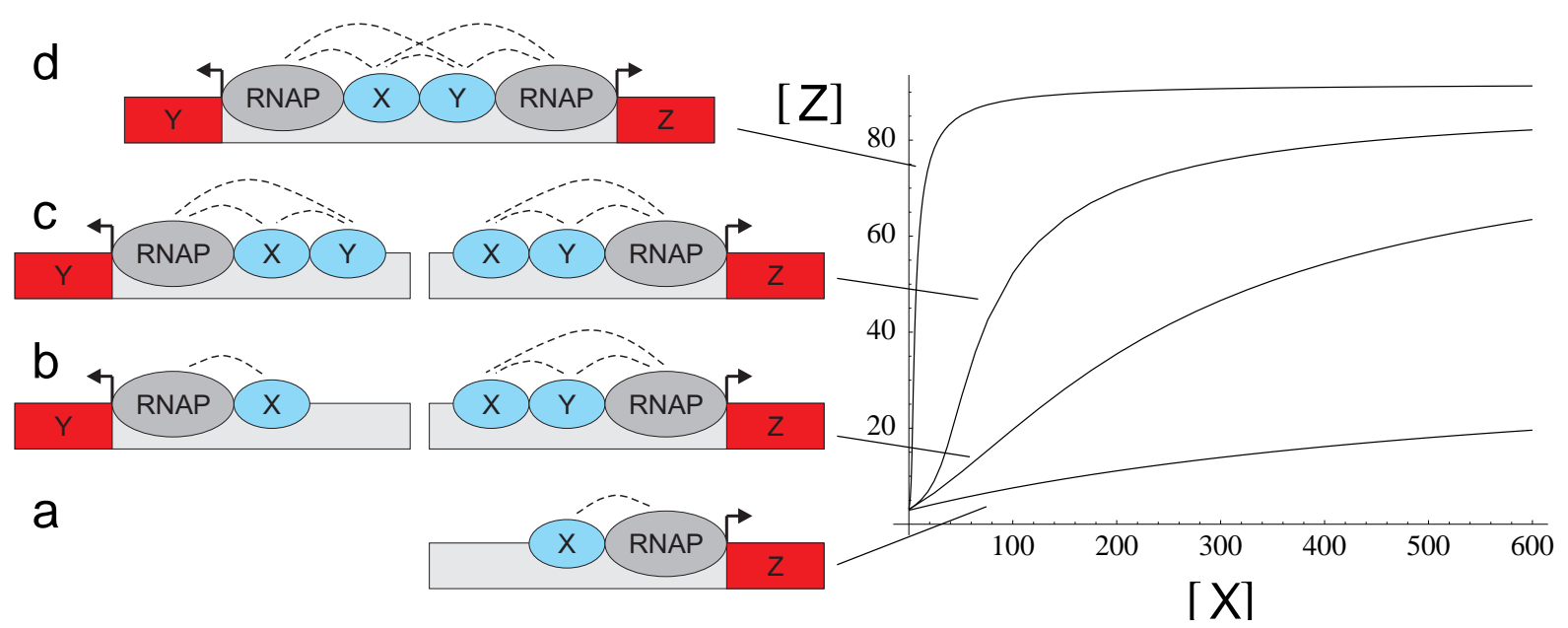

FIG. 6: Response of FFLs as a function of the spatial arrangement of the regulatory elements. A dashed line indicates a weak cooperative interaction of $\approx 3 k_{B} T$, which corresponds to a cooperativity factor $\omega \approx 20$. On the left, the different strucutres: a the expression of gene $\mathrm{Z}$ is activated by the transcription factor $\mathrm{X}$ only; $\mathbf{b}$ a "classical" FFL; $\mathbf{c}$ a FFL with an autoregulatory loop on $\mathrm{Y} ; \mathbf{d}$ a FFL in which the operator regions overlap. On the right, the concentration of the expressed protein $\mathrm{Z}$ in $\mathrm{nM}$ as a function of that of the transcription factor X. Note that structure $\mathbf{d}$ corresponds to structure $\mathbf{c}$ of Fig. 5 of the main text. It is seen that a FFL can act as an amplifier and that overlapping operons can significantly enhance the performance of the amplifier.

Note that this structure corresponds to structure $\mathbf{c}$ of Fig. 5 in the main text.

We have taken $K_{\mathrm{X}}=K_{\mathrm{Y}}=K_{\mathrm{RNAP}}=1000 \mathrm{nM}$, where, for $E$. coli, $1 \mathrm{nM}$ corresponds to roughly one molecule per cell. Note that structure $\mathbf{c}$ of Fig. [5 of the main text corresponds to structure $\mathbf{d}$ of Fig. [6 of the supplementary material.

We have also performed an extra set of calculations, in which there is no direct interaction between the transcription factor Y and the RNA polymerase bound to the promoter for gene Y. For all sets of calculations, we found that the structure in which the operator regions overlap (i.e. structure $\mathbf{c}$ of Fig. 5 of the main text and structure $\mathbf{d}$ of Fig. [6 of the supplementary material) gives the sharpest response. 